\title{
Five genes influenced by obesity may contribute to the development of thyroid cancer through the regulation of insulin levels
}

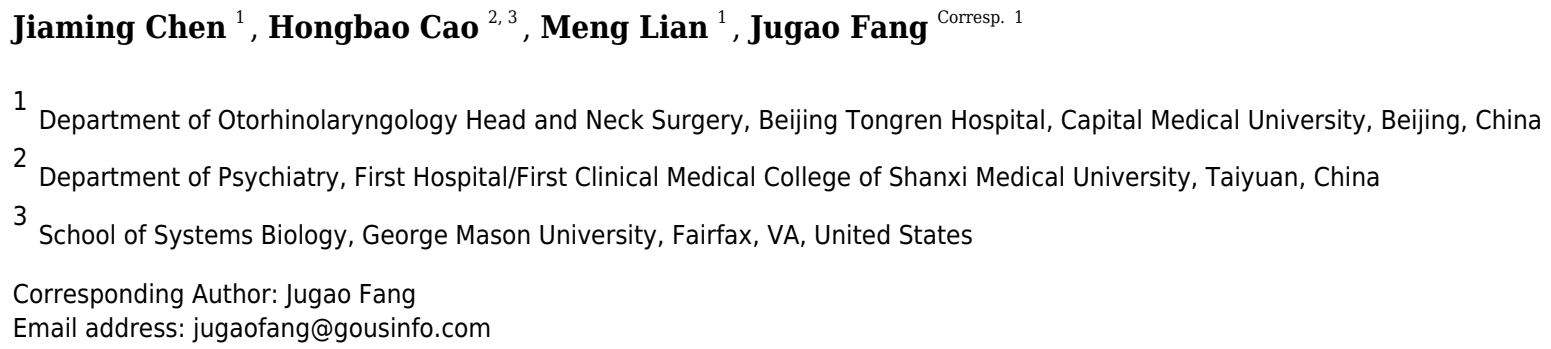

Previous studies indicate that obesity is an important contributor to the proceeding of thyroid cancer (TC) with limited knowledge of the underlying mechanism. Here, we hypothesize that molecules affected by obesity may play roles in the development of TC.

To test the hypothesis above, we first conducted a large-scale literature-based data mining to identify genes influenced by obesity and genes related to TC. Then, a mega-analysis was conducted to study the expression changes of the obesity-specific genes in the case of TC, using 16 independent TC array-expression datasets (783 TC cases and 439 healthy controls). After that, pathway analysis was performed to explore the functional profile of the selected target genes and their potential connections with TC. We identified 1,036 genes associated with TC and 534 regulated by obesity, demonstrating a significant overlap ( $N=176, p$-value $=4.07$-112). Five out of the 358 obesity-specific genes, FABP4, CFD, GHR, TNFRSF11B, and LTF, presented significantly decreased expression in TC patients ( $L F C<-1.44$; and $p$-value $<1 \mathrm{e}^{-7}$ ). Multiple literature-based pathways were identified where obesity could promote the pathologic development of TC through the regulation of these five genes and INS levels. The five obesity genes uncovered could be novel genes that play roles in the etiology of TC through the modulation of INS levels. 
4

\section{Five genes influenced by obesity may contribute to the development of thyroid cancer through the regulation of insulin levels}

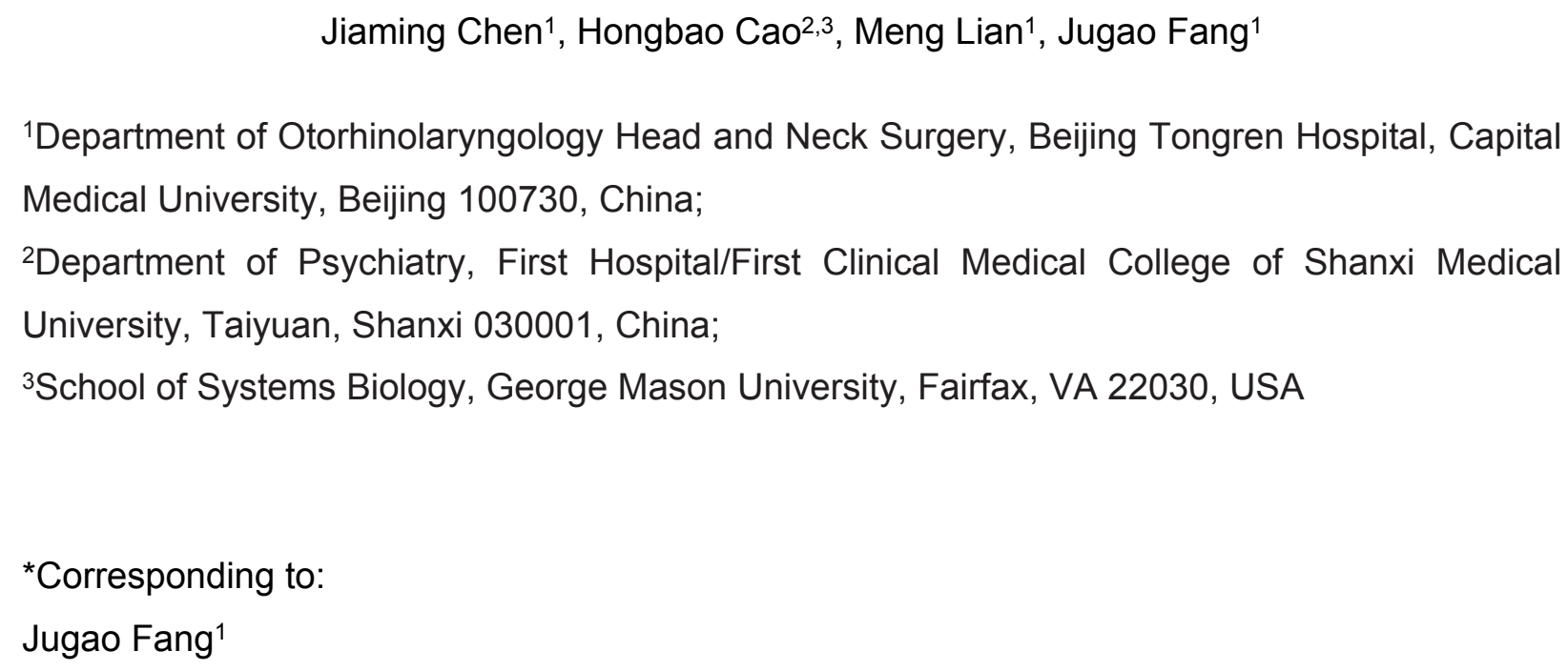

\section{Abstract}


Previous studies indicate that obesity is an important contributor to the proceeding of thyroid cancer (TC) with limited knowledge of the underlying mechanism. Here, we hypothesize that molecules affected by obesity may play roles in the development of TC. To test the hypothesis above, we first conducted a large-scale literature-based data mining to identify genes influenced by obesity and genes related to TC. Then, a mega-analysis was conducted to study the expression changes of the obesity-specific genes in the case of TC, using 16 independent TC array-expression datasets (783 TC cases and 439 healthy controls). After that, pathway analysis was performed to explore the functional profile of the selected target genes and their potential connections with TC. We identified 1,036 genes associated with TC and 534 regulated by obesity, demonstrating a significant overlap $(\mathrm{N}=176, p$-value=4.07e-112). Five out of the 358 obesity-specific genes, FABP4, CFD, GHR, TNFRSF11B, and LTF, presented significantly decreased expression in TC patients (LFC $<-1.44$; and $p$-value $\left.<1 \mathrm{e}^{-7}\right)$. Multiple literature-based pathways were identified where obesity could promote the pathologic development of TC through the regulation of these five genes and INS levels. The five obesity genes uncovered could be novel genes that play roles in the etiology of TC through the modulation of INS levels.

Keywords: thyroid cancer; obesity; data mining; mega-analysis; pathway analysis

\section{Introduction}

Thyroid cancer (TC) develops from the tissues of the thyroid gland and becomes the fastestgrowing cancer of all malignancies (Wolin, Carson, \& Colditz, 2010). Approximately $20 \%$ of all types of cancers might be caused by excessive weight (overweight or obesity) (Wolin, Carson, \& Colditz, 2010). Epidemiologic research suggested that there could be a positive correlation between the increased incidence of both obesity and TC in the past decades (Ogden et al., 2007). The hypothesis has been supported by multiple studies with different methodologies, including cohort study, pooled analysis, and meta-analysis (Engeland et al., 2006; Kitahara et al, 2011; Zhao et al, 2012). However, inconsistent results were represented, which could be due to the unbalanced sex in the TC sample (Meinhold et al., 2010), different sample population regions (Oh, Yoon, \& Shin, 2005), and the lack of adjustment of other influential factors (Meinhold et al., 2010; Engeland et al., 2006).

Nevertheless, many studies have been made to explore the mechanisms underlying the obesity- 
TC relationship, taking the advances made by molecular biologists (Nannipieri et al., 2009; Hard, 1998; Liu et al., 2012; Ozgen et al., 2009; Stassi et al., 2003; lyengar et al., 2017; Park et al, 2016). Some studies showed evidence that regional obesity and a tendency to weight gain were associated with the variations in thyroid function. For example, an increase of triiodothyronine $\left(\mathrm{T}_{3}\right)$ levels was observed in obese subjects (Nannipieri et al., 2009). TSH is the major stimulator of thyrocyte proliferation; the high level of this hormone could be directly involved in thyroid carcinogenesis in obese subjects (Hard, 1998). In addition, multiple genetic and epigenetic alterations of obesity have been reported as pathophysiological important, with many of them also identified as genetic targets for early diagnosis, prognosis or the therapeutic response to the treatment of TC (Liu et al., 2012; Ozgen et al., 2009; Stassi et al., 2003; Iyengar et al., 2017; Park et al., 2016). For instance, separate sets of studies analyzing the pro- and anti-inflammatory cytokines, TNF- $\alpha$, IL-6, and IL-10, which are part of the obesity-associated secretory phenotype, showed their roles in the deterioration or treatment of TC (Liu et al., 2012; Ozgen et al.,2009; Stassi et al. 2003; lyengar et al.2017). However, the mechanism regarding this obesitypromoting-TC relationship remains mostly unclear.

Taken together, these observations indicated the presence of some not-yet discovered connections between obesity and TC. In this study, we attempted to use a system biology approach to identify the not-yet discovered connections between both diseases, including the data mining of disease-gene relation data, the analysis of molecular pathways, and a megaanalysis of existing expression datasets. The integrated analysis of multiple modalities of data has been proven to be an effective way for disease mechanism study (Liu et al., 2019; Zhang et al., 2019; Lian et al., 2019).

\section{Methods and Materials}

This study was organized as follows. First, the large-scale literature-based TC-gene and obesitygene relations data were mined, through which obesity- and TC-genes were identified and compared. Then, a mega-analysis was conducted to test genes that were regulated by obesity but not implicated with TC. After that, a literature-based pathway analysis and a gene set enrichment analysis (GSEA) were performed to identify the potential functional network 
96

97

98

99

100

101

102

103

104

105

106

107

108

109

110

111

112

113

114

115

116

117

118

119

120

121

122

123

124

connecting the selected molecules and TC and the biological profile of these molecules.

\section{Literature-based relation data}

Literature-based genetic relation data was conducted by using Pathway Studio (www.pathwaystudio.com), and results were organized into a supplementary file (Obesity_TC.xlsx). Besides the full lists of genes, we also presented the information of supporting references for each disease-gene relation, including titles of the references and the related sentences where the disease-gene relationships were identified (Obesity_TC $\rightarrow$ TC genes and Obesity_TC $\rightarrow$ Obesity_genes). The information could be used to locate a detailed description of how a candidate gene is associated with obesity and/or TC. To increase the reliability of the obesity affected genes, we selected the obesity-gene relationships with at least three supporting references and with a specific polarity (positive or negative regulation).

\section{Selection of TC-RNA expression Datasets}

We search all TC array-expression datasets available at GEO. After the initial search with keyword 'thyroid cancer', we identified 91 expression datasets for TC. Then the following criteria were applied to fulfill the purpose of this study, including 1) The data organism is Homo sapiens; 2) The data type is RNA expression; 3) The sample size is no less than 10, and 4) the studies are limited to TC cases vs. healthy controls design.

\section{Mega-analysis and target selection}

A mega-analysis was conducted for each of these genes that were regulated by obesity but not associated with TC, using 16 out of 91 TC array-expression datasets from Gene Expression Omnibus (GEO, https://www.ncbi.nlm.nih.gov/geo/). During this step, both the fixed-effect model and random-effects model were employed to study the effect size of the selected genes in a case vs. control expression comparison. The expression log fold change (LFC) was used as the effect size. Results from both models were reported and compared. The heterogeneity of the megaanalysis was analyzed to study the variance within and between different studies. In the case that the total variance $Q$ is equal to or smaller than the expected between-study variance df, the 
125

126

127

128

129

130

131

132

133

134

135

136

137

138

139

140

141

142

143

144

145

146

147

148

149

150

151

152

153

154

155

statistic ISq $=100 \% \times(Q-d f) / Q$ will be set as 0 , and a fixed-effect model was selected for the mega-analysis. Otherwise, a random-effects model was selected. The Q-p represents the probability that the total variance is coming from within-study only. Significant genes from the mega-analysis were reported, which were identified with the criteria as follows: $p$-value $<10^{-7}$ and abs (effect size (log fold change)) $>1$. All analysis was conducted by an individually-developed MATLAB (R2017a) mega-analysis package. We used the term 'mega-analysis' rather than 'meta-analysis' due to the fact that the log-fold changes of each gene were calculated from the original datasets.

\section{Literature-based pathway analysis and GSEA}

For the possible risk genes identified through the expression mega-analysis described above, a literature-based pathway was constructed to identify the connection between the target genes and the TC. The analysis was performed using the 'Shortest Path' module of Pathway Studio (www.pathwaystudio.com). Then all the molecules within the identified networks were tested using a GSEA analysis against the Gene Ontology (GO) terms and Pathway Studio pathways. Significantly enriched pathways and corresponding statistics were reported.

\section{Multiple linear regression analysis}

The MLR model was employed to study the possible influence of three factors on the gene expression change in TC: sample size, population region, and study date. $P$-values and $95 \%$ confidence interval $(\mathrm{Cl})$ were reported for each of the factors.

\section{Results}

\section{Common genes for Obesity and TC}

As presented in the Obesity_TC database, there were 1,036 genes associated with TC and 534 influenced by obesity. A significant overlap of 176 genes was identified for both obesity and TC (Right tail Fisher's Exact test p-value= 4.07e-112), which counts for about one-third of the obesity-regulated genes $(32.96 \%)$. For detailed information on these genes, please refer to Obesity_TC.

\section{The selected gene expression datasets}


156 There were 16 datasets satisfied the selection criteria and were included for the mega-analysis,

157 as shown in Table 1. According to the approach that we acquired the disease-gene relation data 158 (by using Pathway Studio; www. pathwaystudio.com), about 67.04\% of the obesity-genes (358 159 out of 534 genes) have not been reported to have an association with TC. Thus, we tested the 160 expression changes of these 358 genes in the case of TC.

161

162

163

164

\section{Put Table 1 above here.}

\section{Mega-analysis results}

There were five genes (i.g., FABP4, CFD, GHR, TNFRSF11B, and LTF) passed the significance criteria ( $p$-value $<10^{-7}$ and abs $(\mathrm{LFC})>1$ ), which were provided in Table 2 . We presented the mega-analysis results of all obesity-regulated genes in Obesity_TC $\rightarrow$ Mega_Analysis. There were four other genes (TMEM173, PLA2G7, SOD3, and AGTR1) that showed less significance $(p$-value $<7.08 \mathrm{e}-6)$ but also with a big change in terms of LFC (abs (LFC)>1). However, the discussion and analysis here were focused on the five genes that passed the significance criteria. The LFCs of the genes were estimated from the majority of the studies: 15 out of 16 studies. Notably, the Random-effects model was used for CFD and GHR, and the fixed-effect model was selected for FABP4, TNFRSF11B, and LTF. MLR results showed that the age of studies and the sample sizes presented no significant influence on the effect size (LFC) of all five genes except LTF ( $p$-value>0.05), but the sample's population region (country) was a significant factor for all of them ( $p$-value $<0.033$, Table 2$)$.

\section{Literature-based pathway analysis}

To explore the functional association between the five obesity-regulated molecules and TC, we conducted a literature-based functional network analysis and presented in Figure 1 the identified pathways. Results showed that genes GHP, TNFRSF11B, and LTF could be inhibitors of TC, through the stimulation of TC inhibitors or deactivation of TC promoters. In the case of obesity, the activity of these molecules was suppressed. On the contrary, CFD and FABP4 were 
186

187

188

189

190

191

192

193

194

195

196

197

suggested as two facilitators of the pathological development of TC. CFD stimulates INS, which is a promoter of TC. FABP4 inhibits three TC suppressors, including BCL2, PTEN, and PPARG. Notably, obesity activates these two molecules. The pathways revealed in Figure 1 suggested possible mechanisms of the TC-promoting effect of obesity. For the supporting references of the relationships presented in Figure 1, please refer to TC_Obsesity $\rightarrow$ ShortestPath.

\section{Put Figure 1 above here.}

\section{GSEA results}

To understand the functional profile of the 14 genes involved in the pathways presented in Figure 1, we conducted a GSEA against GO terms and Pathway Studio Pathways (www.pathwaystudio.com) and presented the top 10 results in Table 3 . The full list of 31 pathways/GO terms enriched with $p$-value $<0.005$ ( $q=0.005$ for FDR correction) has been listed in TC_Obsesity $\rightarrow$ GSEA. Notably, all the 14 genes were involved in the 31 pathways, and 12 out of 14 were included in the top 10 pathways.

\section{Put Table 3 above here.}

Based on the GSEA results, we analyzed the connection of the five potential TC-genes and nine of their targets presented in Figure 1, in terms of their shared pathways, as shown in Figure 2. The number in a cell represents the number of shared pathways/GO terms by the two corresponding genes, and a number on the diagonal represents the number of pathways enriched by the specific gene. As shown in Figure 2, most of these molecules play roles together with other molecules in multiple pathways, indicating they were functionally connected.

\section{Put Figure 2 above here.}

\section{Discussion}

In this study, we attempted to explore the mechanism underlying the TC-promoting effect of obesity at the genetic level. Towards this purpose, we first used the knowledge-based algorithms 
216 to analyze disease-gene relation data and reveal 176 obesity-regulated genes. Identified genes

217 were also related to TC and utilized to build a common background at the genetic level for the

218 etiology of both obesity and TC. We also uncovered 358 obesity-regulated genes that have not

219 been implicated with TC. To test the potential connection between each of the 358 obesity-

220 regulated genes and TC, we queried and selected the qualified TC-RNA expression datasets

221 from GEO (https://www.ncbi.nlm.nih.gov/geo/), then we conducted a mega-analysis. Five genes

222 were suggested as novel targets for the development of TC, including FABP4, CFD, GHR,

223 TNFRSF11B, and LTF (see Table 2; $p$-value $<10^{-7}$ and LFC<-1.44).

Notably, we used log fold change (LFC) instead of original expression levels for the megaanalysis, which was calculated as the expression levels of the expression level of TC patients over the mean of the expression level of healthy controls, followed by log2 transformation. We assume that, within the same dataset, patients and controls shared a similar background. Thus, by using the LFC, the influence of the background noise was minimized. In addition, we conducted a heterogeneity test for each gene, and a random-effects model was used in the case there was a significant between-study variance such that the study-specific expression variances were taken into account.

FABP4 encodes the fatty acid binding proteins that bind long-chain fatty acids and other hydrophobic ligands. The roles of FABPs include fatty acid uptake, transport, and metabolism (Furuhashi et al., 2015). FABP4 has been showed to induce proteasome degradation of PPARY (Nishina et al., 2017), and decrease the expression of PTEN (Jin et al., 2018) and BCL2 (Yao et al., 2015). Reduced expression of PPARY, PTEN, and BCL2 family proteins have been shown to play critical roles in the pathologic development TC (Copland et al., 2006; Leonardi et al., 2012; Gunda et al., 2017). These pathways in Figure 1 suggested FABP4 as a facilitator of the development of TC through the down-regulation of its inhibitors.

CFD encoded protein adipsin that stimulates the secretion of insulin (Lo et al., 2014), which has been suggested to play roles in the proliferation of TC cells by promoting insulin-like growth factor (Oberman et al., 2015). Therefore, CFD could be a direct promoter of TC. Add together the fact of increased protein levels of FABP4 and CFD in obese patients (Cabré et al., 2012; Kwon et al., 2012) could partially explain the contribution of obesity to TC.

On the contrary, pathways analysis suggested GHP, TNFRSF11B, and LTF as potential TC inhibitors (Figure 1). It has been shown that GHP inhibits the expression of PD-1 (Zhou et al., 
250 2017), which is a TC treatment target (Bi et al., 2019). TNFRSF11B stimulates the secretion of 251 CXCL12 (Benslimane-Ahmim et al., 2011), which was suggested to contribute to TC 252 development by regulating cancer cell migration and invasion (Zhang et al., 2017). Finally, the 253 reduced TGF-beta Type-II receptor (TGFBR2) mRNA was shown to play a role in the 254 pathogenesis of papillary TC (Matoba et al., 1998), while LTF interacts with TGFBR2 to activate 255 TGF- $\beta$ signaling and initiates the formation of TbRIII:TbRII:TbRI complex (Jang et al., 2015).

256 Thereby, decreased levels of GHP, TNFRSF11B, and LTF in obesity could facilitate the 257 pathologic development of TC.

GSEA analysis showed that the five genes (FABP4, CFD, GHR, TNFRSF11B, and LTF) and nine of the downstream target genes mainly played roles in the cell growth related signaling pathways (Table 3). Notably, all these five genes regulate the insulin (INS) levels positively or negatively, while INS level was related to proliferation and carcinogenesis of TC cells (Oberman et al., 2015; Malaguarnera et al., 2017). Our results suggested that obesity may partially affect the pathologic development of TC through its influence on the INS levels.

Moreover, these genes demonstrated a robust functional connection in terms of shared common pathways (Figure 2). The relationship between TC and the nine target genes (INS, BCL2, PTEN, PPARG, PDCD1, CXCL12, EP300, TGFBR2, and CHI3L1) were supported by previous studies (see TC_Obesity $\rightarrow$ ShortestPath), which supports the potential association between the five obesity-regulated genes (FABP4, CFD, GHR, TNFRSF11B, and LTF) and TC.

Nevertheless, this study has several limitations that can be addressed in the future work. First, the connections between the TC and the five obesity genes were suggested by mega-analysis and explored by literature-based pathways analysis. Biologic experiments are needed to test these relationships. Second, due to the lack of space, the discussion was focused on the five genes that passed the significance criteria in the mega-analysis. However, more genes with less significance may also be worthy of inspection (e.g., TMEM173, PLA2G7, SOD3, and AGTR1). Third, further validation of the relationships between the five target genes, insulin, TC, and obesity, can be done using other tools and data sources (e.g., Hetionet v1.0; https://neo4j.het.io/browser/).

\section{Conclusions}

Using the system biology approach, we mined a set of genes influenced by obesity to uncover 
284 five genes (FABP4, CFD, GHR, TNFRSF11B, and LTF) as previously unrecognized contributors

285 to the development of TC. An analysis of functional network built upon these genes points

286 towards INS as a remarkable bridging factor connecting obesity and TC.

287

288 Additional Information and Declarations

289 Competing interests

290 The authors declare that they have no competing interests.

291

292

Acknowledgements

293 Not applicable.

294

295

\section{Author Contributions}

Jiaming Chen collected the original data, analyzed the data, wrote the original paper, and approved the final manuscript.

298

Hongbao Cao developed the study design, revised the original paper, and approved the final manuscript.

Meng Lian developed the study design, revised the original paper, and approved the final manuscript.

Jugao Fang developed the study design, wrote the original paper, and approved the final manuscript.

\section{Data Availability}

All data generated during this study are included in this article and the supplemental files.

\section{Funding}

312 No funding support.

\section{Reference}

Bi Y, Ren X, Bai X, Meng Y, Luo Y, Cao J, Zhang Y, Liang Z. 2019. PD-1/PD-L1 expressions in medullary thyroid carcinoma: Clinicopathologic and prognostic analysis of Chinese population. 
317 Eur J Surg Oncol. 45(3):353-358.

318 Benslimane-Ahmim Z, Heymann D, Dizier B, Lokajczyk A, Brion R, Laurendeau I, Bièche I,

319 Smadja DM, Galy-Fauroux I, Colliec-Jouault S, Fischer AM, Boisson-Vidal C. 2011.

320 Osteoprotegerin, a new actor in vasculogenesis, stimulates endothelial colony-forming cells

321 properties. J Thromb Haemost. 9(4):834-843.

322 Cabré A, Babio N, Lázaro I, Bulló M, Garcia-Arellano A, Masana L, Salas-Salvadó J. 2012.

323 FABP4 predicts atherogenic dyslipidemia development. The PREDIMED study. Atherosclerosis. 324 222(1):229-234.

Copland JA, Marlow LA, Kurakata S, Fujiwara K, Wong AK, Kreinest PA, Williams SF, Haugen BR, Klopper JP, Smallridge RC. 2006. Novel high-affinity PPARgamma agonist alone and in combination with paclitaxel inhibits human anaplastic thyroid carcinoma tumor growth via p21WAF1/CIP1. Oncogene. 25(16):2304-2317.

Engeland A, Tretli S, Akslen LA, Bjørge T. 2006. Body size and thyroid cancer in two million Norwegian men and women. Br J Cancer. 95(3):366-70.

Furuhashi M, Saitoh S, Shimamoto K, Miura T. 2015. Fatty Acid-Binding Protein 4 (FABP4): Pathophysiological Insights and Potent Clinical Biomarker of Metabolic and Cardiovascular Diseases. Clin Med Insights Cardiol. 8(Suppl 3):23-33.

Gunda V, Sarosiek KA, Brauner E, Kim YS, Amin S, Zhou Z, Letai A, Parangi S. 2017. Inhibition of MAPKinase pathway sensitizes thyroid cancer cells to ABT-737 induced apoptosis. Cancer Lett. 395:1-10.

Hard GC. 1998. Recent developments in the investigation of thyroid regulation and thyroid carcinogenesis. Environ Health Perspect. 106(8):427-36.

lyengar NM, Brown KA, Zhou XK, Gucalp A, Subbaramaiah K, Giri DD, Zahid H, Bhardwaj P, Wendel NK, Falcone DJ, Wang H, Williams S, Pollak M, Morrow M, Hudis CA, Dannenberg AJ. 2017. Metabolic Obesity, Adipose Inflammation and Elevated Breast Aromatase in Women with Normal Body Mass Index. Cancer Prev Res (Phila). 10(4):235-243.

Jang YS, Seo GY, Lee JM, Seo HY, Han HJ, Kim SJ, Jin BR, Kim HJ, Park SR, Rhee KJ3, Kim WS4, Kim PH1. Lactoferrin causes $\lg A$ and $\lg G 2 b$ isotype switching through betaglycan binding 
345

346 Jin J, Zhang Z, Zhang S, Chen X, Chen Z, Hu P, Wang J, Xie C. 2018. Fatty acid binding protein

3474 promotes epithelial-mesenchymal transition in cervical squamous cell carcinoma through

348 AKT/GSK3ß/Snail signaling pathway. Mol Cell Endocrinol. 461:155-164.

349

350

351

352

353

354

355

356

357

358

359

360

361

362

and activation of canonical TGF- $\beta$ signaling. 2015. Mucosal Immunol. 8(4):906-17.

Kitahara CM, Platz EA, Freeman LE, Hsing AW, Linet MS, Park Y, Schairer C, Schatzkin A, Shikany JM, Berrington de González A. 2011. Obesity and thyroid cancer risk among U.S. men and women: a pooled analysis of five prospective studies. Cancer Epidemiol Biomarkers Prev. 20(3):464-72.

Kwon EY, Shin SK, Cho YY, Jung UJ, Kim E, Park T, Park JH, Yun JW, McGregor RA, Park YB, Choi MS. 2012. Time-course microarrays reveal early activation of the immune transcriptome and adipokine dysregulation leads to fibrosis in visceral adipose depots during diet-induced obesity. BMC Genomics.13:450.

Leonardi GC, Candido S, Carbone M, Colaianni V, Garozzo SF, Cinà D, Libra M. 2012. microRNAs and thyroid cancer: biological and clinical significance (Review). Int $\mathrm{J}$ Mol Med. 30(5):991-999.

Lian X, Baranova A, Ngo J, Yu G, Cao H. 2019. UGT2B17 and miR-224 contribute to hormone dependency trends in adenocarcinoma and squamous cell carcinoma of esophagus. Biosci Rep. 39(7). pii: BSR20190472.

Liu D, Cao H, Kural KC, Fang, Q, Zhang F. 2019. Integrative Analysis of shared Genetic Pathogenesis by Autism Spectrum Disorder and Obsessive-Compulsive Disorder, Biosci Rep. 39(12). pii: BSR20191942.

Liu Z, Brooks RS, Ciappio ED, Kim SJ, Crott JW, Bennett G, Greenberg AS, Mason JB. 2012. Diet-induced obesity elevates colonic TNF- $\alpha$ in mice and is accompanied by an activation of Wnt signaling: a mechanism for obesity-associated colorectal cancer. J Nutr Biochem. 23(10):120713.

Lo JC, Ljubicic S, Leibiger B, Kern M, Leibiger IB, Moede T, Kelly ME, Chatterjee Bhowmick D, Murano I, Cohen P, Banks AS, Khandekar MJ, Dietrich A, Flier JS, Cinti S, Blüher M, Danial NN, Berggren PO, Spiegelman BM. 2014. Adipsin is an adipokine that improves $\beta$ cell function in diabetes. Cell. 158(1):41-53. 
374 Malaguarnera R, Vella V, Nicolosi ML, Belfiore A. 2017. Insulin Resistance: Any Role in the 375 Changing Epidemiology of Thyroid Cancer? Front Endocrinol (Lausanne). 8:314.

376 Matoba H, Sugano S, Yamaguchi N, Miyachi Y. Expression of transforming growth factor-beta1 and 377 transforming growth factor-beta Type-II receptor mRNA in papillary thyroid carcinoma. 1998. 378 Horm Metab Res. 30(10):624-8.

379 Meinhold CL, Ron E, Schonfeld SJ, Alexander BH, Freedman DM, Linet MS, Berrington de 380 González A. 2010. Nonradiation risk factors for thyroid cancer in the U.S. Radiologic 381 Technologists Study. Am J Epidemiol. 171(2):242-252.

382 Nannipieri M, Cecchetti F, Anselmino M, Camastra S, Niccolini P, Lamacchia M, Rossi M, lervasi 383 G, Ferrannini E. 2009. Expression of thyrotropin and thyroid hormone receptors in adipose tissue 384 of patients with morbid obesity and/or type 2 diabetes: effects of weight loss. Int J Obes (Lond). 385 33(9):1001-6.

386 Nishina A, Itagaki M, Sato D, Kimura H, Hirai Y, Phay N, Makishima M. 2017. The Rosiglitazone387 Like Effects of Vitexilactone, a Constituent from Vitex trifolia L. in 3T3-L1 Preadipocytes. 388 Molecules. 22(11). pii: E2030

389 Oberman B, Khaku A, Camacho F, Goldenberg D. 2015. Relationship between obesity, diabetes 390 and the risk of thyroid cancer. Am J Otolaryngol. 36(4):535-541.

391 Ogden CL, Yanovski SZ, Carroll MD, Flegal KM. 2007. The epidemiology of obesity. 392 Gastroenterology. 132(6):2087-102.

393 Oh SW, Yoon YS, Shin SA. 2005. Effects of excess weight on cancer incidences depending on 394 cancer sites and histologic findings among men: Korea National Health Insurance Corporation 395 Study. J Clin Oncol. 23(21):4742-54.

Ozgen AG, Karadeniz M, Erdogan M, Berdeli A, Saygili F, Yilmaz C. 2009. The (-174) G/C polymorphism in the interleukin- 6 gene is associated with risk of papillary thyroid carcinoma in Turkish patients. J Endocrinol Invest. 32(6):491-494.

Park JW, Han CR, Zhao L, Willingham MC, Cheng SY. 2016. Inhibition of STAT3 activity delays obesity-induced thyroid carcinogenesis in a mouse model. Endocr Relat Cancer. 23(1):53-63.

401 Stassi G, Todaro M, Zerilli M, Ricci-Vitiani L, Di Liberto D, Patti M, Florena A, Di Gaudio F, Di 402 Gesù G, De Maria R. 2003. Thyroid cancer resistance to chemotherapeutic drugs via autocrine 
403

404

405

406

407

408

409

410

411

412

413

production of interleukin-4 and interleukin-10. Cancer Res. 63(20):6784-6790.

Wolin KY, Carson K, Colditz GA. 2010. Obesity and cancer. Oncologist. 15(6):556-65.

Yao F, Li Z, Ehara T, Yang L, Wang D, Feng L, Zhang Y, Wang K, Shi Y, Duan H, Zhang L. 2015. Fatty Acid-Binding Protein 4 mediates apoptosis via endoplasmic reticulum stress in mesangial cells of diabetic nephropathy. Mol Cell Endocrinol. 411:232-242.

Zhang G, Wang W, Huang W, Xie X, Liang Z, Cao H. 2019. Cross-disease analysis identified novel common genes for both lung adenocarcinoma and lung squamous cell carcinoma. Oncol Lett. 18(4):3463-3470.

Zhang S, Wang Y, Chen M, Sun L, Han J, Elena VK, Qiao H. 2017. CXCL12 methylationmediated epigenetic regulation of gene expression in papillary thyroid carcinoma. Sci Rep. $7: 44033$.

Zhao ZG, Guo XG, Ba CX, Wang W, Yang YY, Wang J, Cao HY. 2012. Overweight, obesity and thyroid cancer risk: a meta-analysis of cohort studies. J Int Med Res. 40(6):2041-50.

Zhou M, Yang WL, Aziz M, Ma G, Wang P. Therapeutic effect of human ghrelin and growth hormone: Attenuation of immunosuppression in septic aged rats. 2017. Biochim Biophys Acta Mol Basis Dis. 1863(10 Pt B):2584-2593.

\section{Figure Legends}

Figure 1 The potential pathways connecting the five obesity-regulated genes and thyroid cancer.

Figure 2 Heat map of the shared pathways by the five potential TC-genes and nine of their targets. The number in a cell represents the number of shared pathways/GO terms by the two corresponding genes. 


\section{Figure 1}

The potential pathways connecting the five obesity-regulated genes and thyroid cancer.
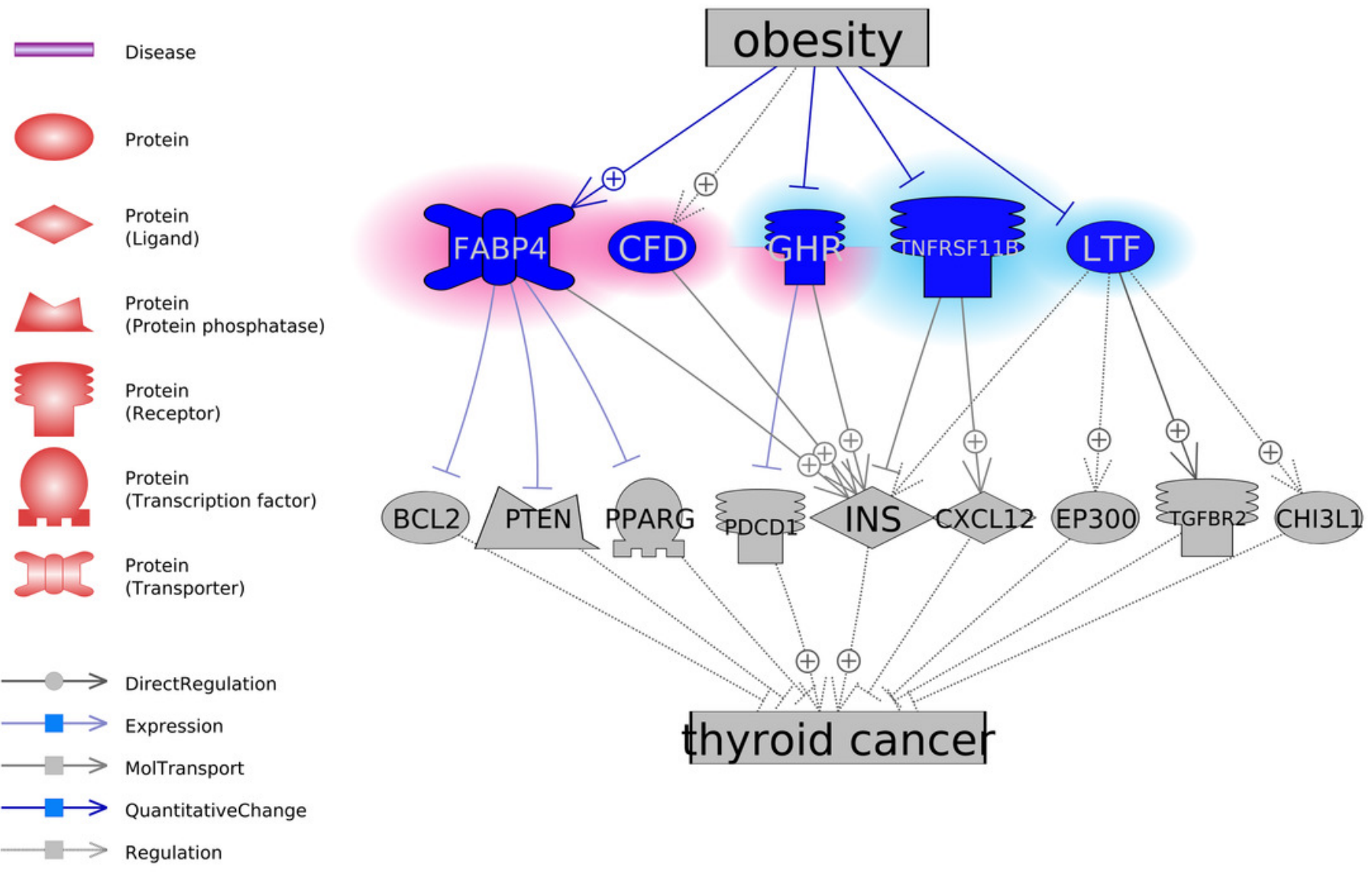


\section{Figure 2}

Heat map of the shared pathways by the five potential TC-genes and nine of their targets.

The number in a cell represents the number of shared pathways/GO terms by the two corresponding genes.

Cross Map

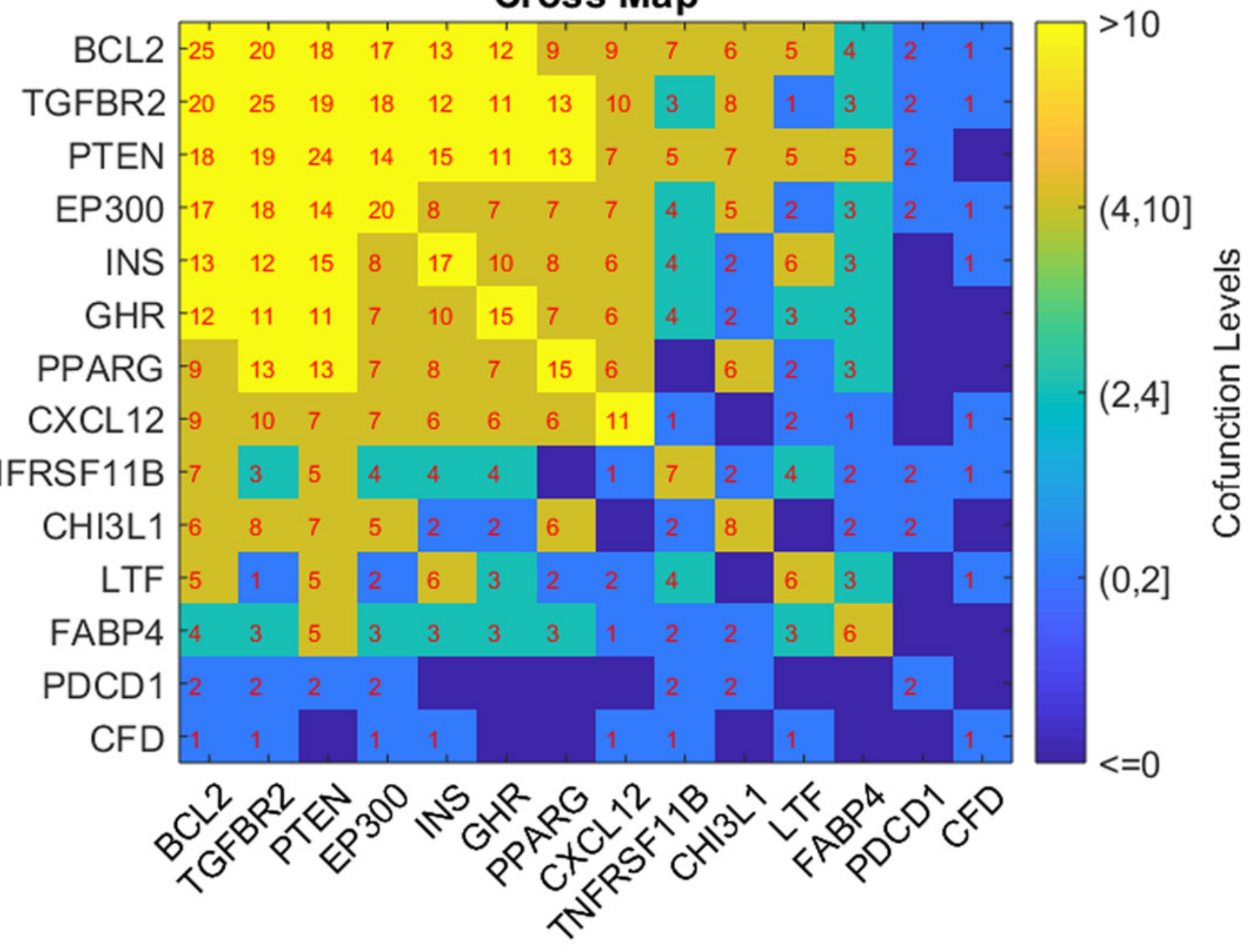




\section{Table 1 (on next page)}

Table 1 The datasets used for gene-TC relation mega-analysis 
1 Table 1 The datasets used for gene-TC relation mega-analysis

\begin{tabular}{|c|c|c|c|c|}
\hline $\begin{array}{c}\text { Dataset } \\
\text { GEOID }\end{array}$ & nControl & nCase & Study Region & Study Age \\
\hline GSE35570 & 51 & 65 & Poland & 4 \\
\hline GSE58545 & 18 & 27 & Poland & 4 \\
\hline GSE58689 & 18 & 27 & Poland & 4 \\
\hline GSE60542 & 34 & 33 & Belgium & 4 \\
\hline GSE65144 & 13 & 12 & USA & 4 \\
\hline GSE39156 & 16 & 48 & Belgium & 6 \\
\hline GSE53157 & 3 & 24 & Portugal & 6 \\
\hline GSE29265 & 20 & 29 & Belgium & 7 \\
\hline GSE33630 & 45 & 60 & Belgium & 7 \\
\hline GSE27155 & 4 & 95 & USA & 8 \\
\hline GSE5364 & 58 & 270 & Singapore & 11 \\
\hline GSE6339 & 135 & 48 & France & 12 \\
\hline GSE9115 & 4 & 15 & USA & 12 \\
\hline GSE3678 & 7 & 7 & USA & 13 \\
\hline GSE6004 & 4 & 14 & USA & 13 \\
\hline GSE3467 & 9 & 9 & USA & 14 \\
\hline
\end{tabular}

2 
Table 2 (on next page)

Table 2 Significant Obesity-genes from mega-analysis for TC 
1 Table 2 Significant Obesity-genes from mega-analysis for TC

\begin{tabular}{|c|c|c|c|c|c|c|}
\hline & Gene Name & FABP4 & CFD & GHR & TNFRSF11B & LTF \\
\hline \multirow{4}{*}{$\begin{array}{c}\text { Mega- } \\
\text { analysis } \\
\text { Results }\end{array}$} & $\begin{array}{c}\text { Using Random } \\
\text { Effects Model }\end{array}$ & 0 & 1 & 1 & 0 & 0 \\
\cline { 2 - 7 } & \#Study & 15 & 15 & 15 & 15 & 15 \\
\cline { 2 - 7 } & $\begin{array}{c}\text { Effect size } \\
\text { (LFC) }\end{array}$ & -1.83 & -1.79 & -1.70 & -1.50 & -1.44 \\
\cline { 2 - 7 } & p-value & $6.4 \mathrm{E}-09$ & $2.03 \mathrm{E}-08$ & $3.61 \mathrm{E}-09$ & $3.24 \mathrm{E}-09$ & $4.17 \mathrm{E}-09$ \\
\hline \multirow{4}{*}{ MLR Results } & nSample & 0.22 & 0.078 & 0.53 & 0.11 & 0.0012 \\
\cline { 2 - 7 } & Country & 0.0019 & $1.01 \mathrm{E}-05$ & $2.24 \mathrm{E}-05$ & 0.033 & 0.029 \\
\cline { 2 - 7 } & studyAge & 0.46 & 0.94 & 0.98 & 0.37 & 0.14 \\
\hline
\end{tabular}

2 


\section{Table 3 (on next page)}

Table 3 The top 10 enriched pathways/GO terms by the 14 genes in regulating pathway identified in Figure 1 
1 Table 3 The top 10 enriched pathways/GO terms by the 14 genes in regulating pathway

2 identified in Figure 1

\begin{tabular}{|c|c|c|c|c|c|}
\hline Name & GO ID & $\begin{array}{c}\text { \# of } \\
\text { Entities }\end{array}$ & Overlap & $\begin{array}{l}\text { Overlapping } \\
\text { Entities }\end{array}$ & $\begin{array}{c}\text { FDR } \\
\text { corrected } \\
p \text {-value }\end{array}$ \\
\hline $\begin{array}{l}\text { regulation of } \\
\text { growth }\end{array}$ & 0040008 & 887 & 8 & $\begin{array}{l}\text { PPARG;BCL2;PTEN;GHR; } \\
\text { INS;CXCL12;TGFBR2; } \\
\text { EP300 }\end{array}$ & 0.00028 \\
\hline $\begin{array}{l}\text { response to } \\
\text { estrogen }\end{array}$ & 0043627 & 155 & 5 & $\begin{array}{c}\text { PPARG;TGFBR2;EP300; } \\
\text { TNFRSF11B;BCL2 }\end{array}$ & 0.00028 \\
\hline $\begin{array}{l}\text { leukocyte } \\
\text { activation }\end{array}$ & 0045321 & 991 & 8 & $\begin{array}{l}\text { BCL2;INS;CHI3L1;CFD; } \\
\text { CXCL12;LTF;TGFBR2; } \\
\text { EP300 }\end{array}$ & 0.00028 \\
\hline $\begin{array}{l}\text { positive } \\
\text { regulation of } \\
\text { growth }\end{array}$ & 0045927 & 354 & 6 & $\begin{array}{c}\text { INS;BCL2;CXCL12;TGFBR2;E } \\
\text { P300;GHR }\end{array}$ & 0.00028 \\
\hline $\begin{array}{c}\text { response to } \\
\text { nutrient } \\
\text { levels }\end{array}$ & 0031667 & 730 & 7 & $\begin{array}{l}\text { PPARG;BCL2;PTEN;GHR; } \\
\text { TNFRSF11B;INS;TGFBR2 }\end{array}$ & 0.00052 \\
\hline $\begin{array}{l}\text { response to } \\
\text { extracellular } \\
\text { stimulus }\end{array}$ & 0009991 & 761 & 7 & $\begin{array}{l}\text { PPARG;BCL2;PTEN;GHR; } \\
\text { TNFRSF11B;INS;TGFBR2 }\end{array}$ & 0.00052 \\
\hline $\begin{array}{l}\text { regulation of } \\
\text { development } \\
\text { al growth }\end{array}$ & 0048638 & 445 & 6 & $\begin{array}{c}\text { BCL2;PTEN;CXCL12;GHR; } \\
\text { TGFBR2;EP300 }\end{array}$ & 0.00052 \\
\hline $\begin{array}{l}\text { response to } \\
\text { glucose }\end{array}$ & 0009749 & 219 & 5 & $\begin{array}{c}\text { INS;BCL2;PTEN;TGFBR2; } \\
\text { EP300 }\end{array}$ & 0.00052 \\
\hline $\begin{array}{l}\text { response to } \\
\text { alcohol }\end{array}$ & 0097305 & 460 & 6 & $\begin{array}{c}\text { PPARG;BCL2;PTEN;GHR; } \\
\text { TGFBR2;EP300 }\end{array}$ & 0.00052 \\
\hline $\begin{array}{l}\text { response to } \\
\text { hexose }\end{array}$ & 0009746 & 227 & 5 & $\begin{array}{c}\text { INS;BCL2;PTEN;TGFBR2; } \\
\text { EP300 }\end{array}$ & 0.00052 \\
\hline
\end{tabular}

